\title{
Role of the colored noise in a FitzHugh-Nagumo system driven by a periodic signal
}

\author{
D. Valenti ${ }^{\star}$, G. Augello, B. Spagnolo \\ Dipartimento di Fisica e Tecnologie Relative, Università di Palermo and \\ CNISM-INFM, Unità di Palermo, Group of Interdisciplinary Physics ${ }^{\sharp}$, \\ Viale delle Scienze, edificio 18, I-90128 Palermo, Italy \\ $\sharp$ URL: http://gip.dft.unipa.it \\ ${ }^{\star}$ e-mail address: valentid@gip.dft.unipa.it
}

\begin{abstract}
We study the role of the fluctuations in the dynamics of a FitzHugh-Nagumo system in the presence both of a periodic driving signal and an additive coloured Gaussian noise with correlation time $\tau$. We investigate the system in two different cases: the equation for the membrane potential contains the noise term (case I), the equation for the recovery variable is noisy (case II). We find that the positions both of minimum of Resonant Activation (RA) and maximum of Noise Enhanced Stability (NES), which appear in the presence of white noise, change as a function of $\tau$, when coloured noise sources are used.
\end{abstract}

Keywords: Neuronal models; Resonant Activation; Noise Enhanced Stability; Colored Noise PACS: 87.19.La, 05.40.-a, 05.45.-a, 02.50

\section{INTRODUCTION}

During these last years the interest in neuronal dynamics increased. The study of this kind of system has been carried out by using the FitzHugh-Nagumo (FHN) model that is a simplified modification of the Hodgkin-Huxley model. Many interesting phenomena can be observed in the presence of fluctuations: modification of detection threshold by manipulation of noisy parameters (FHN model) [1], noise-induced activation and coeherence resonance for suitable noise amplitude (absence of periodic signal) [2], resonant activation for high periodic signals and noise reduction [3], intrinsic stochastic resonance (ISR) in Hodgkin-Huxley neuron and the enhancement of a weak signal by tuning the subthreshold intrinsic oscillation [4], Resonant Activation (RA) and Noise Enhanced Stability (NES) in FHN model [5], dependence of neuron firing on subthreshold periodic signal and additive noise and optimization of the interspike time [6], stochastic resonance maximized in the presence of coloured noise $1 / f^{\beta}$ for $\beta=1$ [7]. In this work, by using coloured noise sources with correlation time $\tau$, we investigate the effects of fluctuations on the FHN dynamics. In particular we study the mean response time (MRT) as a function both of noise intensity and correlation time. 


\section{MODEL}

The stochastic FHN system is described by two coupled equations

$$
\begin{aligned}
\dot{x} & =x-\frac{x^{3}}{3}-y+A \sin \left(\omega t+\varphi_{0}\right)+\zeta_{x} \\
\dot{y} & =\varepsilon(x+I)+\zeta_{y},
\end{aligned}
$$

where $\varepsilon$ is a fixed small parameter which characterizes the recovery process, $A$ and $\omega$ are respectively amplitude and frequency of the external forcing, and $\zeta_{i}(t)(i=x, y)$ are self-correlated noises described by the Ornstein-Uhlenbeck process

$$
\frac{d \zeta_{i}}{d t}=-\frac{1}{\tau} \zeta_{i}(t)+\frac{1}{\tau} \xi_{i}(t)
$$

Here $\xi_{i}(t)$ are statistically independent Gaussian white noises with zero mean and correlation function $\left\langle\xi_{i}(t) \xi_{j}\left(t^{\prime}\right)\right\rangle=\sqrt{\sigma_{i}} \sqrt{\sigma_{j}} \delta_{i j} \delta\left(t-t^{\prime}\right)$ for $i, j=x, y$. In the absence of external driving force there is only one stationary state given by $x_{0}=-I, y_{0}=$ $-I+I^{3} / 3$. The first variable, $x$, provides the membrane potential behaviour, which is characterized by a fast dynamics: after $x$ crosses the threshold value $(x=0)$, rapidly it takes on values below the threshold and a period occurs during which no firing is possible. This condition is connected with the dynamics of the second variable, $y$, characterized by a slow dynamics. Depending on the value of $I$ the stationary point is unstable with stable periodic solution $(|I|<1)$ or stable with all the trajectories converging at this point (attractor) $(|I|>1)[5]$. Here we set $I=1.1$. In this condition a time evolution occurs if the system is driven out of equilibrium. First, we consider the system in deterministic dynamical regime when firing events occur in the absence of noise. This regime takes place when values of amplitude and frequency are chosen inside a certain region of the parameter plane $(\omega, A)$ [5] (see Fig. 1 (a)). Otherwise, for values of $A$ and $\omega$ out of this region no spike occurs in the deterministic dynamics (see Fig. 1 (b)). However, because of the cooperative action of periodic force and noise a stochastic evolution appears that allows the system to fire out of the deterministic firing region (see Fig. 1 (c), (d)).

\section{RESULTS}

We are interested in the mean response time (MRT) of the neuron when external stimulus and colored noise are present. Therefore we don't consider the periodicity of signal. MRT is defined as $\langle T\rangle=1 / N \sum_{i=1}^{N} T_{i}$. Here $T_{i}$ is the first response time (that is, the time for which the first spike occurs) of the $i-t h$ realization and $N$ the total number of realizations. In order to calculate MRT we solve Eqs. $(1,2)$ by performing numerical simulations, with $N=5000$ for case I (the membrane potential is subject to fluctuations) and $N=15000$ for case II (the refractory variable is noisy). We observe Resonant Activation (RA) and Noise Enhanced Stability (NES) phenomena both in cases I and II. 

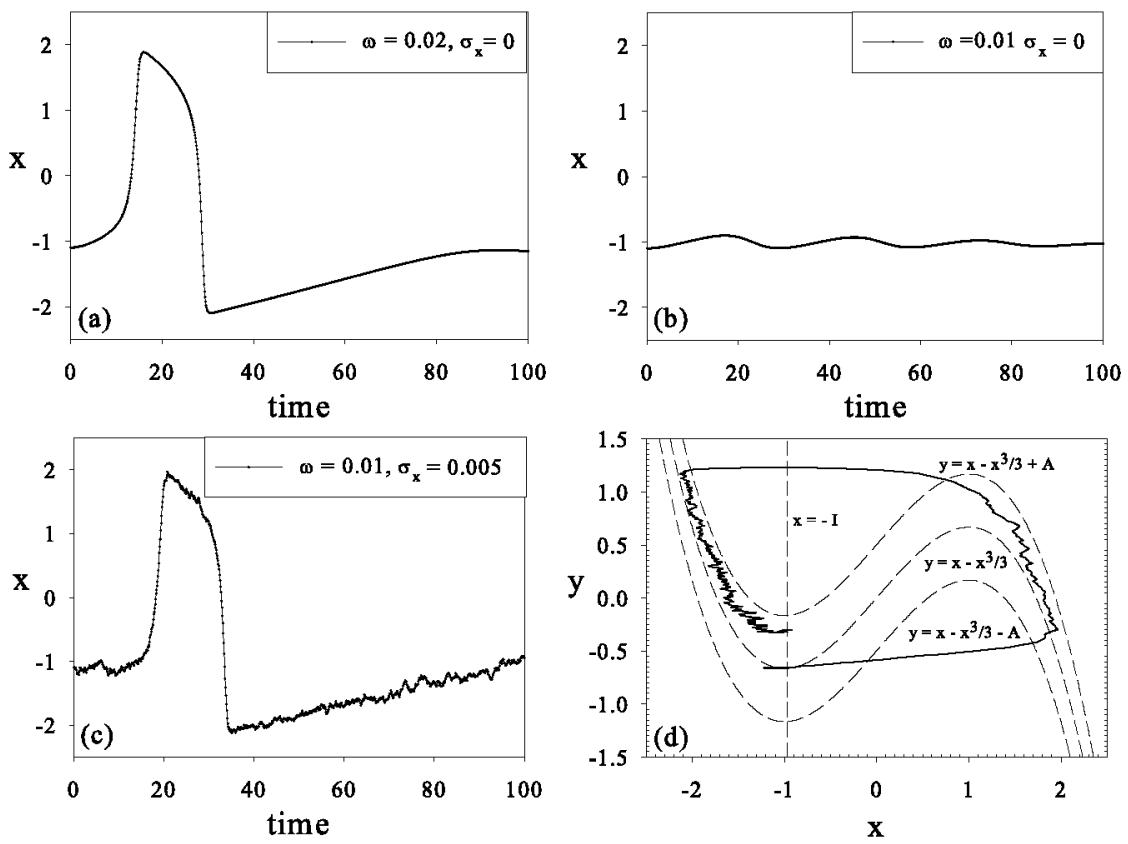

FIGURE 1. (a) The membrane potential $x$ exhibits a spike for $A=0.5$ and $\omega=0.002$ (deterministic firing region). (b) No spike appears (deterministic no-firing region): the values of the parameters $(A=0.5$, $\omega=0.001$ ) don't allow the system to fire. (c) and (d) Stochastic dynamics and corresponding trajectory in the space $(x, y)$ : because of the cooperative action of external signal and fluctuations a stochastic evolution appears that allows the system to fire out of the deterministic firing region.

\section{Resonant activation}

In Fig. 2 we report MRT as a function of $\omega$ for different values both of noise intensities $\sigma_{x}, \sigma_{y}$ and correlation time $\tau$. The minimum of RA depends strongly on the noise intensity. For a low level of noise (see Fig. 2(a), (c)) this minimum is well pronounced when $\tau$ increases (weak suppression of noise effect). However, this phenomenon is more evident at higher noise intensities: for $\sigma_{x}=0.5$ and $\sigma_{y}=0.5$ (see Fig. 2(b), (d)), the behaviour of RA minimum is strongly affected by the correlation time $\tau$. In particular, in case I we find a nonmonotonic behaviour of MRT as a function of $\tau$ in the frequency range $0.1<\omega<1.5$.

\section{Noise Enhanced Stability}

By comparing panels (a) and (b) in Fig. 2 for $\tau=0$ (white noise) and $\tau=5,10$, we observe, in case I (membrane potential is subject to fluctuations), a stabilization effect of the noise, that is, the depth of RA minimum is reduced as the noise intensity increases. 


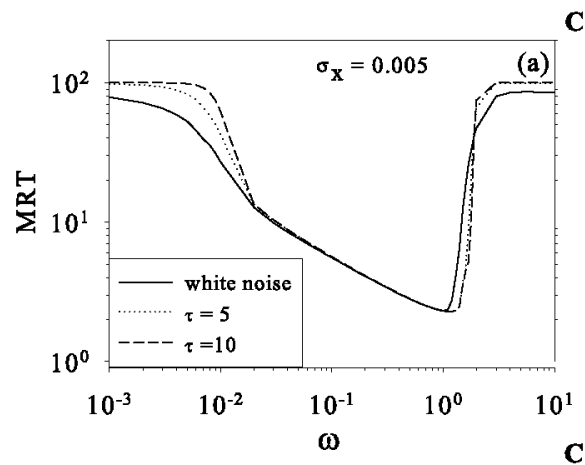

Case I
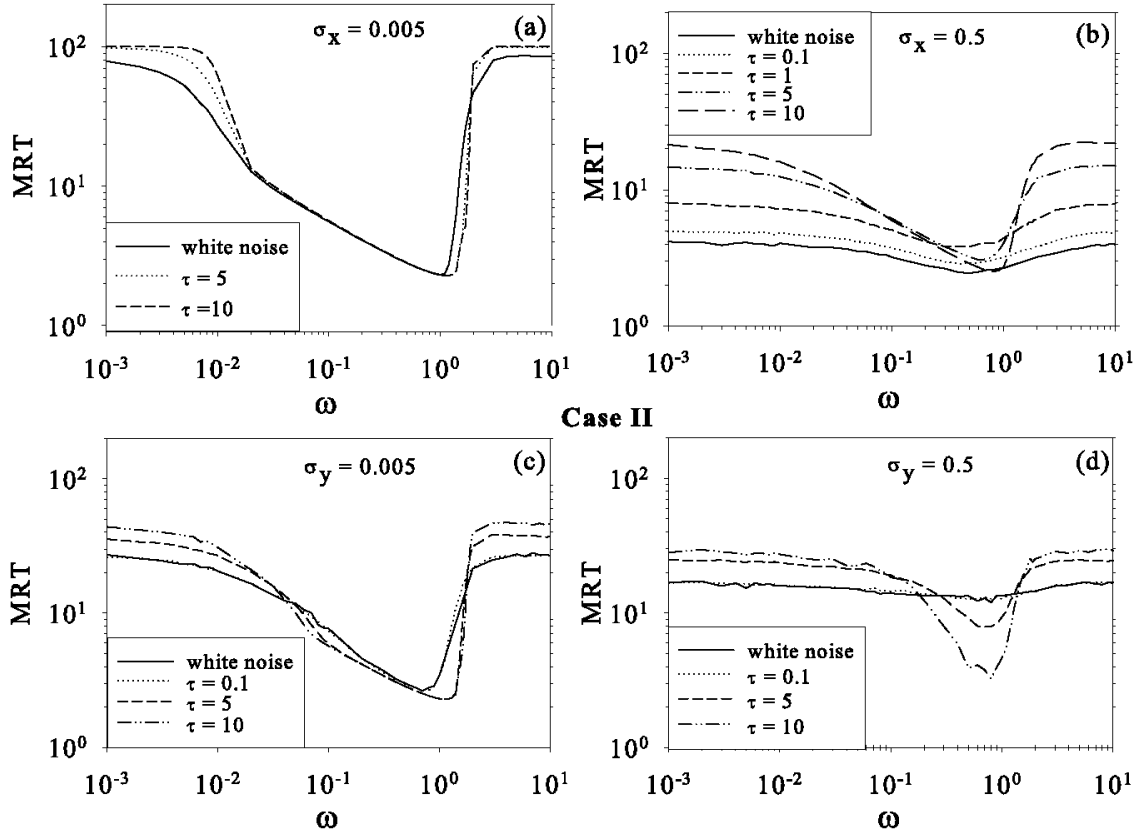

FIGURE 2. (a) and (c) For low noise intensities a slight displacement of the RA minimum is observed as the correlation time $\tau$ increases (weak suppression of the noise effects). (b) and (d) For higher noise intensities the minimum of RA is strongly affected by the value of $\tau$. Both in cases I and II the RA minimum almost disappears for white noise, while it is more pronounced for high values of $\tau$ (strong suppression of the noise effects).

A comparison between panels (c) and (d) of Fig. 2 shows that the same phenomenon is present also in case II (refractory variable is noisy). In order to analyze this increase of mean response time we study the behaviour of MRT around the minimum. Therefore we calculate MRT as a function of the noise intensity for different values of $\tau$, setting $\omega=1$ in case I and $\omega=0.7$ in case II. The results are reported in Fig. 3. In both cases, the strong dependence of MRT on noise intensity is suppressed as correlation time increases.

\section{CONCLUSIONS}

We study the dynamics of a FitzHugh-Nagumo system in the presence of driving periodical force and additive Gaussian coloured noise. We consider two cases: I) fast variable is noisy, II) slow variable is subject to fluctuations. In both conditions RA and NES phenomena appear in the behaviour of MRT. In particular we observe nonmonotonic NES for case I and monotonic NES for case II. Finally, we observe suppression of NES and persistence of RA (efficiency enhancement of neuronal response) as correlation time $\tau$ increases. 

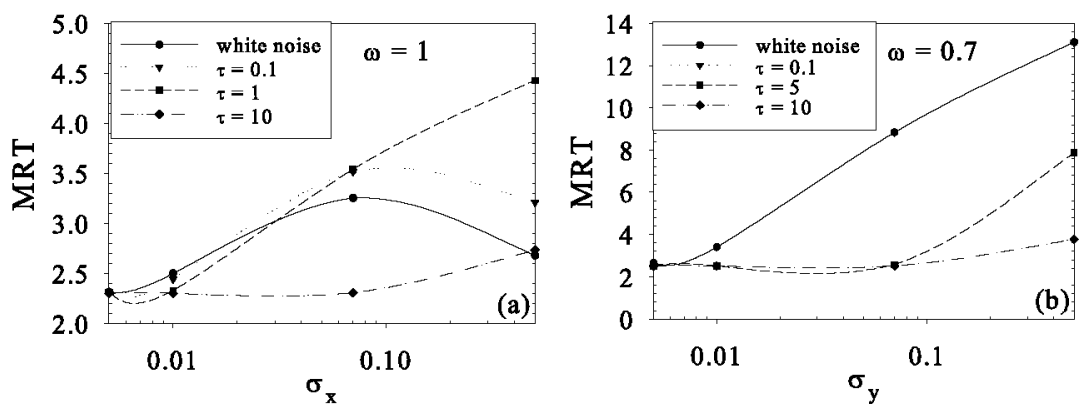

FIGURE 3. (a) Case I. Nonmonotonic behaviour of MRT as a function of white noise intensity: in the presence of coloured noise this effect disappears as correlation time increases. (b) Case II. Monotonic increase of MRT as a function of white noise intensity: this behaviour is suppressed for strongly correlated noise.

\section{ACKNOWLEDGMENTS}

This work was supported by MIUR and CNISM-INFM.

\section{REFERENCES}

1. X. Pei, K. Bachmann, and F. Moss, Phys. Lett. A 206, 61-65 (1995).

2. A. S. Pikovsky, and Jürgen Kurths, Phys. Rev. Lett. 78, 775-778 (1997).

3. A. L. Pankratov, and M. Salerno, Phys. Lett. A 273, 162-166 (2000).

4. D. T. W. Chik, Y. Wang, Z. D. Wang, Phys. Rev. E 64, 021913(1-6) (2001).

5. E. V. Pankratova, A. V. Polovinkin, B. Spagnolo, Phys. Lett. A 344, 43-50 (2005).

6. A. Longtin, D. R. Chialvo, Phys. Rev E 81, 4012-4015 (1998).

7. D. Nozaki, Y. Yamamoto, Phys. Lett. A 243, 281-287 (1998). 